\title{
La «mamma» di Guglielmo VIII Paleologo: Maddalena Panattieri da Trino, terziaria OP (1443-1503)
}

The «mother» of Guglielmo VIII Paleologo: Maddalena Panattieri from Trino tertiary $O P$ (1443-1503)

\section{Gabriella ZARRI}

Storia Moderna

Facoltà di Lettere e Filosofia

Università di Firenze

zarri.gabriella@gmail.com

\begin{abstract}
After a brief excursus on the Italian «living saints» who acquired a reputation for sanctity between the second half of the fifteenth and sixteenth centuries and a clarification of the historiographical concept of «living saints», the essay presents the hitherto unexplored case of the Dominican tertiary Maddalena Panatieri da Trino (1443-1503). While living in a small town in Monferrato, a rich land located in the Piedmont region, Maddalena was reported to the Marquis Guglielmo VIII Paleologo for having prophesied his victory in a tournament held in France. Later venerated by the prince, who considered her a mother, the life of the blessed Magdalene was composed by Girolamo da Milano OP following the example of the hagiographic legend of Blessed Osanna of Mantua, after the passage of Monferrato to the Gonzaga dukedom in the thirties of the sixteenth century.
\end{abstract}

Keywords: Marquisate of Monferrato; Living saint; Maddalena da Trino: hagiography and worship.
Astratto: Dopo un breve excursus sulle «sante vive» italiane vissute tra il secondo Quattrocento e il Cinquecento e una precisazione del concetto storiografico di «sante vive», il saggio presenta il caso fino ad ora non approfondito, della terziaria domenicana Maddalena Panatieri da Trino (1443-1503). Mentre viveva in un piccolo centro del Monferrato, ricca terra situata nella regione del Piemonte, Maddalena si segnalò al marchese Guglielmo VIII Paleologo per aver profetizzato la sua vittoria in un torneo tenutosi in Francia. Venerata in seguito dal principe, che la considerò una madre, la vita della beata venne composta da Girolamo da Milano op sull'esempio della leggenda agiografica della beata Osanna da Mantova, dopo il passaggio del Monferrato al ducato dei Gonzaga negli anni Trenta del cinquecento.

Parole chiave: Marchesato del Monferrato; Santa viva; Maddalena da Trino: agiografia e culto.

Conosciuta in vita e venerata dopo la morte, Maddalena da Trino è stata scarsamente ricordata dalla cronachistica coeva e dalla letteratura critica. La sua vicenda è tuttavia meritevole di una attenzione particolare in quanto rappresenta una manifestazione precoce di quel fenomeno storico che si è convenuto definire 
con l'espressione di «sante vive» ${ }^{1}$. Come ho avuto occasione di ricordare recentemente $^{2}$, i fatti che hanno consentito di evidenziare nel contesto italiano una peculiarità non riscontrata altrove per contemporaneità ed estensione del fenomeno ci conducono alla speciale temperie culturale e religiosa della fine del secolo XV, contrassegnata dall'inizio delle guerre d'Italia e dalla tragica esecuzione di Girolamo Savonarola. Instabilità politica, predicazione profetico-apocalittica, credenza nell'astrologia favoriscono il ricorso dei principi al consiglio di maghi e indovini, ma anche di religiosi ritenuti santi.

È certamente vero che la profezia politica ha una consolidata tradizione fin dal periodo basso medievale in diversi paesi europei e vede protagoniste anche le donne ${ }^{3}$, e forse non è ancora sufficientemente studiata, nonostante il recente approfondimento, l'influenza esercitata da Brigida di Svezia ${ }^{4}$, Caterina da Siena ${ }^{5}$, Giovanna d'Arco ${ }^{6}$, sulla cultura e i comportamenti delle donne che le assunsero come modello fin dall'inizio del secolo XV, tuttavia non è arbitrario considerare il caso italiano talmente peculiare da divenire paradigmatico.

La discesa del re francese Carlo VIII in Italia nel 1494 per rivendicare a sé con le armi il possesso del regno di Napoli in mano aragonese dà inizio a un conflitto che interessa le maggiori potenze europee, ma ha come campo di battaglia per un cinquantennio esclusivamente l'Italia e vede implicati tutti i maggiori principati

1 Gabriella ZARri, Le sante vive. Per una tipologia della santità femminile nel primo Cinquecento, in Annali dell'Istituto Storico Italo-Germanico in Trento, 6 (1980), pp. 372-445, ora in EAD., Le sante vive. Cultura e religiosità femminile nella prima età moderna, Torino, 1990; EAD., Les prophètes de cour dans l'Italie de la Renaissance, in Mélanges de l'Ecole Française de Rome - Moyen Age, t. 102 (1990-2), pp. 649-675; EAD., Potere carismatico e potere politico nelle corti italiane del Rinascimento, in Agostino Paravicini Bagliani e André Vauchez (eds.), Poteri carismatici e poteri informali, Palermo, 1992, pp. 164-180.

2 Gabriella ZARRI, Legittimare il principe: principi rinascimentali italiani e la promozione del culto dei propri santi domenicani, in Viliam Stefan DÓCI OP, Gianni FESTA OP (eds.), Fra trionfi e sconfitte. La «politica della santità» dell'Ordine dei Predicatori, Roma, 2021, in corso di stampa.

3 André VAUCHEZ, Saints, prophètes et visionnaires: le pouvoir surnaturel au Moyen Age, Paris, 1999; Alessandra BARTOLOMEI ROMAGNOLI, Caterina e Robina: profezia e antiprofezia al tempo del grande scisma d'Occidente, in EAD., Santità e mistica femminile nel Medioevo, Spoleto, 2013, pp. 630-656.

4 Maria H. OEN (ed.), A companion to Birgitta of Sweden and her Legacy in the Later Middle Ages, Leiden, Boston, 2019; Sanctity and female authorship: Birgitta of Sweden \& Catherine of Siena, Maria H. OEN and Unn FalKeID (eds.), New York, London, 2020.

5 Virgo digna coelo: Caterina e la sua eredità: raccolta di studi in occasione del $550^{\circ}$ anniversario della canonizzazione di santa Caterina da Siena (1461-2011), a cura di Alessandra BARTOLOMEI ROMAGNOLI, Luciano CINELLI, Pierantonio PIATTI, Città del Vaticano, Libreria Editrice Vaticana, 2013.

6 Philippe CONTAMINE, Olivier BOUZY, Xavier HÉLARY, feanne d'Arc: histoire et dictionnaire, Paris, 2012; Philippe CONTAMINE, De feanne d'Arc aux guerres d'Italie : figures, images et problèmes du XV Siècle, Orléans, 1994. 
e le piccole signorie che si trovano in balia di continui mutamenti. Dalla discesa di Carlo VIII, che le profezie apocalittiche identificano come il nuovo Ciro che purificherà la chiesa, Girolamo Savonarola inizia la sua avventura politica, tragicamente conclusa con il rogo. Negli stessi anni donne penitenti e devote, per lo più terziarie domenicane che si ispirano al modello di vita di Caterina da Siena, acquistano fama di santità presso la popolazione, che le ammira e le venera come «sante vive», mentre le autorità cittadine cercano di trattenerle nelle proprie aree urbane concedendo loro dimora e privilegi. Al manifestarsi dei doni carismatici di queste donne, come estasi, visioni, stigmatizzazioni, ha inizio un sistematico interesse di principi e signori nel contattare ciascuna di queste, con l'intento di condurle nel proprio stato - come è il caso di Ludovico il Moro che tenta inutilmente di condurre a Milano la terziaria domenicana Stefana Quinzani ${ }^{7}$, o quello di Ercole I d'este che opera con successo per sottrarre la stigmatizzata Lucia Brocadelli da Narni ai Viterbesi ${ }^{8}$ - e presentarle come carismatiche vicine alla corte e come potenti protettrici del popolo.

Anche in questo caso non mancano precedenti illustri di santi vicini alle corti. Si pensi ad esempio all'eremita Francesco di Paola, condotto in Francia dalla Calabria da Luigi XI nel 1482 e trattenuto dai successori presso di loro fino alla morte del religioso, avvenuta nel $1507^{9}$. Di Francesco di Paola tuttavia si apprezzavano soprattutto i poteri taumaturgici, mentre nel caso delle «sante vive» si ricercano piuttosto i carismi profetici e le penitenze espiatrici che consentano ai principi di presentare il santo, o più spesso la santa, come voce di Dio, come vittima sacrificale per i peccati del popolo e come baluardo della città.

Non soltanto donne, ma prevalentemente tali, le figure carismatiche legate alle corti italiane tra il 1494 e il 1555 sono, a mia conoscenza, complessivamente $15^{10}$. Di queste 13 sono donne (Maddalena Panatieri da Trino, Colomba Guadagnoli da Rieti, Lucia Brocadelli da Narni, Osanna Andreasi da Mantova, Stefana

7 ZARRI, Le sante vive. Cultura e religiosità... [ver n. 1], p. 96.

$8 \quad$ Ibid., pp. 57-61 e E. Ann MATTER e Gabriella ZARRI, Una mistica contestata. La Vita di Lucia da Narni (1476-1544) tra agiografia e autobiografia. Con l'edizione del testo, Roma, 2011.

9 Saint Francois de Paule et les Minimes en France de la fin $d u X V^{e}$ au XVIIT siècle, textes réunis et présentés par Benoist PIERRE e André VAUCHEZ, Tours, 2010; L'eremita Francesco di Paola viandante e penitente. Atti del III Convegno internazionale di studio (Paola, 14-16 settembre 2000), Roma, 2006; Commende, Osservanza e riforma tra Italia, Francia e Spagna. Atti del Convegno di studi (Roma, 22-24 novembre 2007), Roma, 2014.

10 Gabriella ZARRI, Profeti di corte nell'Italia del Rinascimento, in Daniel BORNSTEIN, Roberto RUSCONI (eds.), Mistiche e devote nell'Italia tardomedievale, Napoli, 1992, pp. 209-236, ora in EAD., La chiesa dei principi e delle città tra medioevo e prima età moderna, Spoleto, 2021, pp. 267-294. 
Quinzani da Soncino, Elena Duglioli da Bologna, Chiara Bugni da Venezia, Veronica Negroni da Binasco, Arcangela Panigarola da Milano, Margherita Molli da Russi, Gentile Giusti da Ravenna, Caterina Mattei da Racconigi, Caterina de' Ricci, fiorentina che vive a Prato) e 2 sono di sesso maschile (il bolognese Arcangelo Canetoli, eremita a Gubbio e il fiorentino Michelangelo Bonaventura Pini, romito camaldolese). Tra le donne prevalgono le terziarie domenicane, che sono ben $7^{11}$, e che si caratterizzano rispetto alle altre mistiche e devote per il forte accento savonaroliano dei loro discorsi e delle loro rivelazioni. Come ha messo efficacemente in luce Tamar Herzig ${ }^{12}$, le imitatrici di Caterina diventano anche la «voce» di Savonarola. Nei tre decenni successivi la morte del profeta, il savonarolismo, soffocato a Firenze dal restaurato regime Mediceo, esce dalla città toscana attraverso gli esiliati e le mistiche visionarie e si diffonde nei diversi centri dell'Italia pontificia e padana per bocca dei predicatori e delle sante di corte che, pur a fianco dei principi regnanti, sollecitano la riforma della chiesa e invocano la giustizia sociale. Lo stretto legame che unisce terziarie devote e confessori domenicani dell'Osservanza concorre a configurare la rappresentazione culturale della «santa viva» che ha il suo prototipo nella beata Osanna da Mantova, la cui vita, composta da fra Francesco Silvestri, esce a stampa in latino e in volgare negli anni 1506 e $1507^{13}$.

Sarebbe troppo lungo ricordare le singole carismatiche analizzate e approfondite. Qui intendo soltanto ribadire che, pur in presenza di numerosi casi analoghi in diversi paesi europei, che vengono progressivamente alla luce facendo emergere, specialmente in Castiglia e Andalusia, un pullulare di visionarie conosciute e sostenute dalla regina Isabella e dal Card. Cisneros ${ }^{14}$, le «sante vive» italiane costituiscono un gruppo strettamente legato ad un preciso contesto storico e culturale ed hanno un sostanziale significato politico. In un periodo di estrema

11 Maddalena Panatieri da Trino, Colomba Guadagnoli da Rieti, Lucia Brocadelli da Narni, Osanna Andreasi da Mantova, Stefana Quinzani da Soncino, Caterina Mattei da Racconigi, Caterina de' Ricci.

12 Tamar HERZIG, Savonarola's women: visions and reform in renaissance Italy, Chicago, London, 2008.

13 Francesco SILVESTRI, Beatae Osannae Mantuanae de tertio habitu ordinis fratrum predicatorum uita per fratrem Franciscum Siluestrum Ferrariensem eiusdem ordinis \& uitae regularis professorem edita, Impressum Mediolani, per Petrum Martirem de Mantegatiis dictum Cassanum, 1506 die $20 \mathrm{Au}-$ gusti; ID., La vita e stupendi Miraculi dela gloriosa virgine Osanna Mantuana del Terzo ordine de Frati Predicatori (Impressa in Milano, apresso di Alexandro Minutiano, a sexdeci di Ianuario. 1507).

14 Si veda Alessandra BartolomeI Romagnoli (eds.), Sante vive in Europa (secoli XV-XVI)/Santas vivas en Europa (siglos XV-XVI), numero monografico di Archivio italiano per la storia della pietà, XXXIII, 2020 e Catálogo de Santas Vivas (1400-1550): Hacia un corpus completo de un modelo bagiográfico femenino diretto da Rebeca SANMARTÍN BASTIDA http://catalogodesantasvivas.visionarias.es/index.php/P\%C3\%A1 gina_principal\#Proyecto [consultato il 15 marzo 2021]. 
instabilità istituzionale, precarietà economica e disagio sociale, il «santo di corte» forniva protezione al popolo con il suo potere taumaturgico e al tempo stesso conferiva al principe una legittimità sacrale tanto più importante nel caso di un potere contestato o contrastato. La prospettiva politica, qui sinteticamente ricordata, resta a mio parere uno degli aspetti che consente di interpretare il fenomeno delle «sante vive» quale manifestazione strettamente legata ad un periodo circoscritto e a una temperie culturale irripetibile, condizionata, come si è detto, dal desiderio di dare legittimità sacrale a signorie e principati nuovi o contestati, non paragonabili con le corone di Francia, Inghilterra e Spagna che avevano una solida legittimazione dinastica e sacrale. Come ha recentemente rilevato Sofia Boesch Gajano, i diversi studi su queste figure femminili hanno inteso mettere a fuoco l'interpretazione di una esperienza religiosa peculiare, non estensibile ai living saints che si riscontrano in ogni paese e in ogni tempo ${ }^{15}$.

Il caso della terziaria di Trino Vercellese si presta efficacemente a rappresentare l'aspetto tipologico e la valenza politica della santa viva legata alla corte. Maddalena nacque a Trino nel 1443 e apparteneva alle famiglie Panatieri (o Pannatieri) per parte di padre e Fondazucca per parte di madre. Lo attesta per la prima volta, attingendo probabilmente a una tradizione familiare, il padre domenicano Pietr'Antonio Fondazucca, autore di una vita della beata stampata a Milano nel $1644^{16}$. Mistica e visionaria, Maddalena avrebbe profetizzato la vittoria del marchese di Monferrato Guglielmo VIII Paleologo (1420-1483) in un torneo tenutosi in Francia, le stragi delle guerre d'Italia e la pace successiva ad opera di «un gran capo cristiano» ${ }^{17}$. La profezia della vittoria in Francia creò un legame di particolare favore del principe con la terziaria domenicana, la cui vicenda, in parte ignorata, merita una più ampia analisi. Occorre anzitutto considerare le fonti storiche, letterarie e icnografiche che ci danno notizie della donna devota, riconosciuta beata dalla chiesa per culto ab immemorabili nell'anno $1827^{18}$.

15 Sofia BOESCH GAJANO, Le «sante vive»: un'invenzione storiografica da brevettare, in Concetta BIANCA, Anna SCATTIGNo (eds.), Scritture carismi istituzioni: percorsi di vita religiosa in età moderna. Studi per Gabriella Zarri, Roma, 2018, pp. 361-366.

16 P. Fondazucca, Vita della beata Maddalena Pannatieri da Trino Professa del Terz'Ordine del Patriarca S. Domenico... Dedicata a' molt' illustri signori Sindici di Trino, Milano, Francesco Vigone, 1702 (I ediz. 1644, come si deduce dalla lettera dedicatoria datata 1644).

17 Ibid., p. 49.

18 Angelico IsZAK, Panatieri, Maddalena, beata, in Bibliotheca Sanctorum, vol. 10, Roma, 1998, coll. 77-78; Acta sanctorum, Auctarium ad diem XIV Octobris, De B. Magdalena de Panateriis Virgine, Tertii Ordinis S. Dominici, Tridini in Dioecesi Vercellensi, Bruxelles, 1965-1970 (Ripr. facs. dell'ed.: Anversa, poi Bruxelles, 1643-1940), pp. 168-178; Renato VASCONI, La donna che incarnò la gioia: storia della beata Maddalena da Trino, Alba, 1972. 
La più antica testimonianza della santità della donna ci è giunta dal domenicano Ambrogio Taegio che, scrivendo nel secondo decennio del Cinquecento, fa riferimento ai miracoli avvenuti al suo sepolcro ${ }^{19}$. La più antica leggenda agiografica della terziaria trinese, composta dal padre Girolamo da Milano ${ }^{20}$ intorno agli anni 1534-35, riferisce puntualmente le estasi e i miracoli della carismatica, dando particolare enfasi al suo amore per la chiesa e per la città. Pochi anni dopo la composizione della leggenda, un autorevole pittore locale, Ottaviano Cane (1495-1576), dipinge per i domenicani di Trino un matrimonio mistico di santa Caterina in cui figura una religiosa in abito domenicano, ravvisabile in Maddalena $^{21}$. Nella seconda metà del Cinquecento la fama di santità della mistica vercellese è attestata non solo a livello locale ma anche nell'intero ordine domenicano, come testimonia Serafino Razzi che trae le notizie da lui narrate da un dipinto allora esposto nella chiesa dell'Incoronata di Ancona ${ }^{22}$.

Il culto della terziaria prosegue nei secoli successivi, pur senza far emergere Maddalena da Trino dall'insieme delle altre carismatiche che avevano avuto fama di santità in vita. Essendo stata fin qui ricordata senza ulteriori approfondimenti

19 Sacra Rituum Congregatione E.mo et R.mo Domino Cardinali de Somalia (Giulio Maria Cavazzi della Somaglia) Praefecto et ponente, Tridentinen, seu Ordinis Praedicatorum pro confirmatione cultus ab immemorabili tempore praestiti B. Magdalenae de Panateriis Tertii Ordinis S: Dominici... Memoriale cum Summario, Animadversiones R.P.D. Promotoris Fidei, et Responsiones, Romae MDCCCXXVII, ex Typographia Reverendae Camerae Apostolicae, p. 3. La Cronaca del domenicano Ambrogio Taegio, rimasta manoscritta, è conservata ora nell'Archivio Generale dell'Ordine dei Predicatori (=AGOP) a Roma, Convento di Santa Sabina. Sullo scrittore e la sua opera v. Silvia Nocentini, Le Grazie nell'opera di Ambrogio Taegio in Stefania BUGANZA, Marco Giuseppe RAININI (eds.), Il convento di Santa Maria delle Grazie a Milano. Una storia dalla fondazione a metà del Cinquecento. Atti del Convegno di Studi (Milano, 22-24 maggio 2014), Memorie domenicane, $n$. s. 47 (2016), pp. 79-102.

20 Nativo di Barlassina, ma detto da Milano, forse per l'usanza di riferirsi alla città più vicina, fu eccellente predicatore, secondo quando indica Matteo CEREGHINO OP, La Beata Maddalena Panatieri da Trino, Genova, 1927, p. 187. La figura di questo religioso domenicano resta ancora oscura e la vita di Maddalena da lui composta è rimasta manoscritta e priva di studi critici. Recentemente tuttavia è stata pubblicata una edizione modernizzata della vita antica, ad uso divulgativo: GIROLamo Da Milano, La beata Maddalena da Trino. Maddalena donna di Dio, donna del Rinascimento, Trino, Tipografia AGS, 2005.

21 Sul pittore v. Giovanni Romano, Cane, Ottaviano, in Dizionario biografico degli italiani (=DBI), vol. 17 (1974), https://www.treccani.it/enciclopedia/ottaviano-cane_(Dizionario-Biografico)/ [consultato il 24.12.2020]. Il dipinto si trova ora a Torino alla Galleria Sabauda, mentre una riproduzione si può vedere nel testo sopra citato: GIROLAMO DA MILANO, La beata Maddalena da Trino: vita manoscritta della beata Maddalena da Trino, p. 32.

22 Serafino RAZZI, Vite de i santi, e beati del sacro Ordine de' frati predicatori, cosi buomini, come donne. Con aggiunta di molte vite, che nella prima impressione non erono. In Firenze, nella stamperia di Bartolomeo Sermartelli, 1588, pp. 150-151. 
nelle indagini storiche recenti ${ }^{23}$, ci pare interessante ripercorrere brevemente l'origine e lo sviluppo del culto della Trinese, cercando di comprendere i motivi del faticoso e tardivo riconoscimento di santità.

Bisogna partire dal territorio e dal tempo in cui si manifesta il potere carismatico della giovane domenicana ed esaminarne il profilo nell'opera agiografica composta un trentennio dopo la sua morte.

Trino Vercellese era un centro importante del marchesato del Monferrato, territorio del Piemonte che si estendeva tra le province di Alessandria, Asti e Vercelli. Prevalentemente collinare, con appendici anche nella zona padana, il marchesato venne costituito nel secolo $\mathrm{X}$ per la cessione di questo territorio ad Aleramo e ai suoi discendenti da parte dell'imperatore Ottone I. Allora incolto e in parte paludoso, il terreno venne sottoposto a intensa coltivazione e a creazione di vigneti e risaie così da renderlo altamente fruttifero. Nel 1303, per estinzione degli Aleramici, il marchesato passò ai Paleologi, famiglia di origine greca che mantenne il suo dominio fino al 1533, anno in cui passò ai Gonzaga di Mantova.

Nella sua storia pluricentenaria, il marchesato raggiunse il culmine della propria potenza e cultura nel secolo XV, dapprima sotto Giovanni IV ${ }^{24}$, e poi durante il dominio di Guglielmo VIII Paleologo, marchese dal 1464 al 148425. Con la costruzione del palazzo marchionale, Casale divenne il centro dell'amministrazione del marchesato, e con la erezione di nuovi edifici sacri e l'introduzione di religioni legate all'Osservanza lombarda, il borgo si preparava a divenire diocesi e città ${ }^{26}$. Fin dal primo Quattrocento, infatti, i Paleologi promossero l'ingresso nel loro stato degli Ordini mendicanti, fino a quel momento largamente minoritari rispetto ai più antichi ordini benedettini. Il primo convento domenicano del marchesato fu quello di Santa Caterina di Alessandria di Trino, fondato nel 1403

23 Antonella PERIN, Carla SOLARINO, Casale e il territorio nella prima metà del secolo XV: architetture tra perdite e sopravvivenze, in Carlo ALETTO, Antonella PERIN (eds.), Casale Monferrato, una capitale per il territorio: le premesse: da Teodoro 2. a Giovanni 4. (1404-1464): atti del Convegno, Genova, 2019, p. 102.

24 Ibid., pp. 71-185.

25 Aldo SETTIA, Guglielmo VIII, Marchese del Monferrato, in DBI, vol. 60, 2003, https://www.treccani. it/enciclopedia/guglielmo-viii-marchese-di-monferrato_\%28Dizionario-Biografico\%29/ [consultato il 26.12.2020].

26 Enrico LuSSO, La committenza architettonica dei Marchesi di Saluzzo e di Monferrato nel tardo Quattrocento: modelli mentali e orientamenti culturali, in Lucia CORRAIN, Francesco P. DI TEODORO (eds.), Architettura e identità locali, vol. I; indici a cura di Emanuela VAI, Firenze, 2013, pp. 423438; ID., I conventi del principe. Fondazioni dei Predicatori e strategie urbane nel Monferrato paleologo, in Gli ordini mendicanti e la citta'. I frati predicatori, a cura di Diego LANZARDO, Bruno TARICCO (eds.), Cherasco, 2009 («Miscellanea di Storia degli insediamenti», I), pp. 89-120: 89-97. 
su iniziativa di una donatrice, affiancata dal sostegno finanziario e dal consenso costante dei vari principi che si avvicendarono fino al compimento dell'opera e alla consacrazione della chiesa, avvenuta nel $1458^{27}$.

Principato di medie dimensioni, racchiuso tra il ducato di Savoia, la repubblica di Genova e lo stato di Milano, dal punto di vista istituzionale il marchesato del Monferrato era un feudo soggetto all'imperatore, trasmissibile soltanto per via maschile e quindi soggetto a possibili rischi, non infrequenti a quei tempi, di estinzione della dinastia. Dal punto di vista politico i Paleologi privilegiarono i rapporti culturali e di alleanza con la vicina Francia, a cui si rivolsero anche per alleanze matrimoniali, ma, da abili condottieri, si destreggiarono tra le diverse potenze a seconda delle convenienze del momento.

A Guglielmo VIII, come si è detto, si deve la scelta di Casale come sede della corte, a cui si collegò non soltanto lo sviluppo economico, la trasformazione urbanistica del borgo, l'erezione della diocesi, ma anche l'animazione culturale della residenza marchionale. A questo proposito chiamò a corte illustri umanisti e istituì a Casale una scuola di grammatica e di retorica, coltivando soprattutto i contatti con l'umanesimo lombardo ${ }^{28}$; stimolò e appoggiò lo sviluppo dell'arte tipografica ${ }^{29}$; chiamò a Casale i domenicani Osservanti promuovendo la fondazione del convento di San Domenico ${ }^{30}$.

In questo contesto, nel 1443 nacque e condusse la sua vita una fanciulla del luogo che, secondo l'agiografo, era molto devota a santa Caterina da Siena e volle essere terziaria domenicana ancora molto giovane. Assidua frequentatrice della chiesa di Santa Caterina di Alessandria dei domenicani Osservanti, era vista frequentemente andare in estasi durante la preghiera; talvolta rivolgeva esortazioni ai novizi e a quanti si fermavano per ascoltare le sue parole; era sempre pronta ad aiutare le persone che avevano bisogno o si rivolgevano a lei per preghiere.

Un giorno, mentre era a Trino con alcune compagne, comunicò loro che in quel momento il principe, che si trovava in Francia, aveva battuto in torneo due cavalieri. Si seppe successivamente che il fatto annunciato era effettivamente avvenuto nel giorno e orario predetto. Quando Guglielmo VIII, al suo ritorno

27 Ibid., pp. 100-109.

28 Paolo Rosso, La cultura delle èlites marchionali nella Casale della prima metà del Quattrocento, in Carlo Aletto, Antonella PERIN (eds.), Casale Monferrato, una capitale per il territorio... [ver n. 23], pp. 31-69.

29 ID., La politica culturale dei Paleologi fra Quattro e Cinquecento e i suoi riflessi nell'editoria del marchesato, in Magda BALBONI (ed.), Trino e l'arte tipografica nel XVI secolo, Novara, 2014, pp. 71-90.

30 ID., La cultura delle èlites marchionali... [ver n. 28], pp. 47-50. 
dalla Francia, apprese la predizione di Maddalena, manifestò alla donna grande onore e gratitudine. Vedremo dopo quanto scrive testualmente l'agiografo a questo proposito. Ora è interessante soffermarci sul rapporto di reciproca stima e fiducia instauratosi tra il principe e la giovane carismatica che, pur senza vivere presso la corte, può effettivamente considerarsi una figura che entra a far parte della «familia» del principe.

Poiché i fatti descritti avvengono in data imprecisata, ma prima della morte di Guglielmo VIII avvenuta nel 1483, potremmo chiederci se nella seconda metà del secolo XV esistevano altre figure femminili ricercate e venerate da principi o signori feudali e se le loro doti mistiche erano assimilabili a quelle delle più tarde «sante vive». In effetti proprio in terra padana, nella città di Parma e nelle signorie limitrofe si erano manifestati due carismi sostenuti da famiglie aristocratiche che ne promuovevano la conoscenza e la canonizzazione. Il primo e più antico di questi culti è quello prestato dai Sanvitale, conti di Fontanellato, nei confronti della beata Orsolina Veneri da Parma, mistica che operava e profetizzava nel periodo dello scisma della chiesa e che morì nei primi anni del secolo $\mathrm{XV}^{31}$. Il secondo è invece contemporaneo alla vita e vicenda di Maddalena da Trino, ed è rappresentato da Simona della Canna, umile mistica protetta da Pietro Maria Rossi, conte di Berceto e San Secondo, che nel 1476 fece erigere per lei un sepolcro in cattedrale con una epigrafe che ne attestava la vita santa e ne chiedeva la canonizzazione ${ }^{32}$. Non diversamente, in territorio lombardo l'umile contadina Veronica da Binasco, conversa nel monastero di S. Marta di Milano, ebbe molteplici rapporti con gli Sforza, non esitando a esortare Ludovico il Moro a porre rimedio ai peccati della sua corte ${ }^{33}$.

31 Tra i molti studi su Orsolina da Parma, v. Renate BLumEnfELD-KosInsKI, Bruce L. VENARDE, Two Women of the Great Schism: The Revelations of Constance de Rabastens by Raymond de Sabanac and the Life of Ursulina of Parma by Simone Zanacchi, Toronto, 2010. Su Orsolina v. inoltre Renate BLuMENFELD-KosinsKi, Poets, Saints, and Visionaries of the Great Schism (1378-1417), University Park, Pennsylvania 2006, pp. 86-89; Alessandra BARTOLOMEI RomagnOLI, Santità e mistica femminile nel Medioevo cit., particolarmente pp. 641-642, 646-647; Antonella DEGL'INNOCENTI, Un'opera da riscoprire: le Visiones della beata Osolina da Parma, in Hagiographica, XXVII, 2020, pp. 379-402.

32 Gabriella ZARRI, Profeti di corte nell'Italia del Rinascimento... [ver n. 10], ora in EAD., La chiesa dei principi e delle città... [ver n. 10], p. 273; Gianluca BATTIONI, Aspetti della politica ecclesiastica di Pier Maria Rossi, in Letizia ARCANGELI, Marco GENTILE (eds.), Le signorie dei Rossi di Parma tra XIV e XVI secolo, Firenze, 2007, pp. 106-107; Giuseppa Z. ZANICHELLI, La committenza dei Rossi: immagini di potere fra sacro e profano, Ibid., p. 211.

33 Cfr. Angeliche visioni: Veronica da Binasco nella Milano del Rinascimento, a cura di Alessandra BARTOLOMei Romagnoli, Emore PaOli, Pierantonio Piatti, Firenze, 2016. 
Simona dalla Canna può certamente considerarsi, per il suo rapporto con il principe, un caso precoce di «santa viva», ma non viene descritta come tale dagli agiografi, mentre Maddalena da Trino che vive ed opera ancora negli anni della vicenda savonaroliana può essere ritratta dal suo primo agiografo con gli accenti infiammati delle contemporanee mistiche domenicane. Altrettanto può dirsi dell'agostiniana Veronica da Binasco la cui prima vita a stampa è composta dal domenicano Isidoro Isolani con l'intento di presentarla ai conquistatori francesi del ducato di Milano ${ }^{34}$.

Ritornando al caso di Maddalena Panatieri da Trino, occorre esaminare il testo che ne tramanda vita, profezie e miracoli. Come ho detto precedentemente, il primo racconto agiografico della beata di Trino è composto da un domenicano Osservante lombardo, Girolamo da Milano, che non è ricordato nei maggiori repertori biografici degli scrittori domenicani. La sua stessa opera doveva aver avuto scarsa circolazione all'interno dell'ordine, dal momento che il famoso e informato Serafino Razzi, che pure è a conoscenza di un culto prestato alla beata Maddalena, non attinge dal testo agiografico le notizie che trasmette su di lei ma le deduce da un dipinto conservato nel convento domenicano di una regione distante dal luogo di origine della terziaria vercellese.

Mai dato alle stampe, neppure il manoscritto originale di Girolamo da Milano pare essersi conservato. Nell'archivio del convento domenicano di Trino, ora a Torino, si conservano tre copie molto tarde dell'autografo cinquecentesco.

La prima copia, apparentemente la più antica, ha per titolo: Vita manoscritta della B. Maddalena da Trino, composta dal P. F. Girolamo da Milano Domenicano, con in fine alcune attestazioni in favore della Beata. Copia non autentica né originale. Fogli decinove. A dispetto del titolo, questa copia reca un notevole numero di testimoni, tra cui due notai, che affermano che la vita di Girolamo da Milano è autentica e che le cose riferite sono vere (pp. 17v-19r). Il manoscritto è inoltre preceduto da una Dedica al Lettore del padre Fondazucca, il primo biografo della Beata. Ciò consente di dedurre che la copia si deve collocare alla metà del secolo XVII e che forse era stata preparata per la stampa ${ }^{35}$.

34 Isidoro ISOLANI, Inexplicabilis mysterii gesta beatae Veronicae virginis praeclarissimi monasterii sanctae Marthae urbis Mediolani. Sub obseruatione regulae diui Augustini, Mediolani, apud Gotardum Ponticum impressorum, 1518 die III Aprilis.

35 Archivio Storico Provinciale dell'Ordine dei Predicatori - Sezione di Torino (=ASPOP-T.) Trino, S. Caterina V. M., R 1. Vita manoscritta della B. Maddalena da Trino, composta dal P. F. Girolamo da Milano Domenicano, con in fine alcune attestazioni in favore della Beata. 
Il secondo manoscritto è una copia ottocentesca di una recensione leggermente diversa della vita di fra Girolamo da Milano, ed è preceduta da un foglio che ne descrive la provenienza da un privato, che l'aveva ricevuta dopo la soppressione napoleonica del convento ${ }^{36}$.

Il terzo manoscritto invece è una copia ottocentesca del primo manoscritto e reca il titolo: Copia fedele della Vita manoscritta della B. Maddalena da Trino, composta dal P. F. Girolamo da Milano Domenicano, con in fine alcune attestazioni in favore della Beata. Tale manoscritto è inoltre arricchito da un notevole numero di note apposte da domenicani del secolo XIX ${ }^{37}$.

Nelle citazioni che seguono mi attengo a quanto asserisce il primo manoscritto, indicato come il più antico. Il profilo della beata Maddalena come Madre del principe si configura in relazione alla prima attestazione di carisma profetico manifestato dalla terziaria Trinese. In un anno imprecisato, Guglielmo VIII Paleologo si trovava in Francia e sosteneva un torneo con due cavalieri. Risultò vincitore e questo difficile certame fu antivisto da Maddalena:

Essendo il marchese Guglielmo ${ }^{38}$ in Franza di vintidue anni combatte con dui et hebbe la vittoria, et la verginella disse in quell'hora et punto, ch'egli havea hauto la vittoria della giostra per grazia de Dio e per l'orationi de' suoi servi et devoti. E così poi venne la nova che la vittoria era seguita in quell'ora et punto istesso ${ }^{39}$.

Saputo l'accaduto, il Marchese mostrò la propria gratitudine donando a Maddalena una veste nuova e la chiamò Madre, non mancando di andare ad incontrarla ogni volta che ritornava a Trino:

Dove il Signore, poi che fu ritornato, la vesti di novo, e la chiamò sempre per Madre, e non veniva mai a Trino, che subbito non la dimandasse dicendo: «Dove è mia Madre?». Essa gli predisse molte cose che gli dovevano accadere, et così è stato vero il tutto ${ }^{40}$.

Dopo questa prima manifestazione profetica, la fama della terziaria trinese si consolidò e l'agiografo riferisce le molte visioni e rivelazioni di Maddalena. Per

36 Ibid., preceduta da un foglio con l'avvertimento: Importante.

37 Ibid., Cartolario n. 2.

38 Nell'interlinea superiore è aggiunta, erroneamente, la parola Gonzaga

39 ASPOP-T., Trino, S. Caterina V. M., R 1. Vita manoscritta cit., f. 6r. Nessuno dei biografi della terziaria trinese rilevano l'incongruenza della visione, dovuta all'età dei protagonisti. Guglielmo VIII aveva 22 anni nel 1442, mentre la «verginella» sarebbe nata nel 1443, secondo il dato fornito dal Fondazucca.

40 Ibidem. 
prima cosa predisse le tribolazioni che dovevano riversarsi sull'Italia, e in particolare sopra la Lombardia, in conseguenza dei peccati:

Predisse anco molte tribulationi, che dovevano venire sopra la Christianità, massime sopra la Lombardia, cioè peste, guerra, distruttioni di persone, svergognamento di donne maritate, stupri di fanciulle. Et legi di monache claustrali caciate da monasteri, svergognate pubblicamente, con molte altre tribulationi che dovevano venire alla Santa Chiesa et Christiani per li gran peccati che erano al mondo, massime nelli Capi ${ }^{41}$.

Nel narrare la visione avuta da Maddalena nella chiesa dei domenicani, nella cappella detta dei Marchesi, l'agiografo rimarca il fetore dei peccati degli ecclesiastici e l'ira di Dio sul suo popolo, e molto più forte si evidenzia l'accento savonaroliano che egli conferisce a quelle pagine:

Perché non gli era né Charità né giustitia, ma ogni cosa era per il contrario et a pena si raccordavano de Dio, onde sua Maestà divina voleva mostrar il flagello della sua giustitia alla Christianità, dopo il quale dovea seguir grand' unione et pace per la presa d'un gran Capo Christiano. Hebbe questa visione nella Chiesa di Santa Chaterina Martire ch' è delli frati di San Domenico in una capella presso il choro, a man destra, chiamata la capella di Santa Margarita del Signore o del Crucifisso, nella quale è dipinta la passione di Nostro Signore Giesù Christo. Et ciò fu nel giorno della Pentecoste. Fu rapita in spirito e vidde Dio Padre che pareva star turbato ad udir l'anima che pregava e non la volere esaudire; e lei perseverava in pregare per la Christianità, onde gli rispose il Padre Eterno e disse: $\ll \mathrm{O}$ figliola mia carissima, non vedi che la puzza vuole che siano puniti? $»^{42}$.

Nel constatare la fermezza con cui il Signore intendeva punire la cristianità, Maddalena, secondo uno stereotipo presente in tutte le scritture agiografiche relative alle «sante vive», supplica di risparmiare almeno il suo popolo e la sua città e non cessa di pregare e invitare i credenti alla conversione:

Et Dio all'hora gli rispose: «Figliola mia, altro non vorrei che penitenza et emendatione. Ma non vedi tu quanta superbia regni in ogni stato e grado?» $\mathrm{Di}$ rapine, d' ingiustitie et estorsioni, e di turpissime operationi ogni cosa è piena. Non vedi che hanno voltà la faccia da me, e sono immersi nel fetente lezzo de' suoi peccati, di maniera che la mia giustitia non gli può più sopportare? All'hora l'anima prostrata in terra repplicò con singulti: «Deh Signore prego la tua im-

41 Ibid., ff. 6r e v.

42 Ibidem. 
mensa bontà che almeno voglia far misericordia alla patria mia e al popolo mio». Alla quale rispose Iddio: «O figliola mia cara, sebene gli suoi peccati sieno troppo grandi, ad ogni modo per amor tuo et per tante orationi che sono fatte da alcuni miei servi ancora aspettarò per qualche giorno». Dove stava tutta di mala voglia, e pensando sopra gli flagelli ch' haveva preparato Iddio alla Christianità, massime all'Italia, per gran dolore non si poteva rihavere, mandando lagrime e singulti in grandissima copia; pure raccordandosi di quel Capo Christiano per il quale si dovea far pace et unione, alquanto si rihebbe, e tornata in se stessa, piangendo e sospirando cominciò a dire: «Guai a te Italia! Guai a te Italia! ${ }^{43}$.

Non mancano nella leggenda agiografica della terziaria di Trino i racconti di miracoli, né le espressioni profetiche prive di significato politico, ma non c'è dubbio che il rapporto della mistica con il principe conferisca alla Maddalena di Trino una fisionomia del tutto analoga a quella delle «sante vive» dell'Italia centrale e padana.

Pochi anni dopo la morte di Maddalena, sempre nel Piemonte, Claudio di Savoia-Racconigi, detentore del feudo di Racconigi, promuoveva l'insediamento dei domenicani Osservanti e proteggeva la terziaria Caterina Mattei, da loro presentata come santa. Il legame del principe nei confronti della donna devota è documentato dal primo testamento redatto da Claudio nel 1517, in cui figura un vitalizio per la «figliola» Caterina, aumentato di altri benefici nel codicillo redatto il 27 ottobre $1520^{44}$. Anche in questo caso il principe instaura con la carismatica un rapporto di tipo familiare, chiamandola «figlia».

Come abbiamo visto nel caso delle due «sante vive» piemontesi, la reciproca stima e legittimazione che si instaura tra principe e donna devota assume la rappresentazione del legame familiare. Le Madri tendono tuttavia a prevalere sulle Figlie, almeno nell'area geografica e culturale più affine al Monferrato: il marchesato, poi ducato di Mantova.

Come accennato precedentemente, la leggenda agiografica di Maddalena da Trino è composta negli anni 1534-35, subito dopo il passaggio del marchesato di Monferrato al dominio del duca di Mantova, avvenuto nel 1533 per la morte del giovane principe Giovanni Giorgio privo di eredi e l'assunzione del governo da parte di Federico II Gonzaga, marito di Margherita Paleologo, unica superstite della dinastia. Nello stato dei Gonzaga vi era ormai una lunga tradizione di Madri

43 Ibid., ff. 6v-7r.

44 ASPOP-TO, Racconigi, S. Vincenzo, Pergamene non inventariate, VI: Testamento (trascritto) di Claudio di Savoia, e Codicillo a parte, relativo ai domenicani, in pergamena (1517); VIII, Codicillo 1520 . 
carismatiche: le terziarie domenicane Osanna Andreasi, beatificata nel $1515^{45}$, e suor Stefana Quinzani morta nel 1530 e beatificata nel $1740^{46}$. Le diverse vite a stampa della Andreasi ${ }^{47}$ avevano certamente influenzato la Vita di Maddalena da Trino composta da Girolamo da Milano, che forse si proponeva l'intento di offrire una continuità carismatica domenicana alla corte di Mantova, in quel periodo alla ricerca di un'altra protettrice celeste.

Per comprendere il relativo ritardo nel riconoscimento del culto di Maddalena da Trino bisogna forse pensare alla mancanza di un supporto autorevole alla sua morte. Maddalena muore infatti nel 1503, nella stagione in cui fiorivano molte altre carismatiche viventi. Nel marchesato di Monferrato le condizioni politico-culturali erano mutate. Nel 1508 Guglielmo IX Paleologo aveva sposato Anna di Alençon, cognata di Margherita di Angoulême, che si mostrò legata all'ordine domenicano promuovendo l'erezione in Casale del monastero femminile di Santa Caterina, ove poi si ritirò a vivere negli ultimi anni della sua vita ${ }^{48}$. Ella condivideva con le altre principesse italiane e francesi di quel periodo la fiducia e l'interesse verso donne carismatiche considerate sante; nel 1519 invitò alla sua corte una «santa viva» bolognese, Elena Duglioli ${ }^{49}$, e volle farla conoscere alle amiche delle corti vicine, come Isabella Este Gonzaga e Lucrezia Borgia ${ }^{50}$. Anche la figlia di Anna, Margherita, che andò sposa nel 1531 a Federico II Gonzaga,

45 Su Osanna Andreasi la bibliografia recente è vastissima: Rodolfo SIGNORINI, Rosanna GOLINELli BERTO (eds.), Osanna Andreasi da Mantova 1449-1505: la santità nel quotidiano, Mantova, 2005; Renata CASARIN (ed.), Osanna Andreasi da Mantova 1449-1505: immagine di una mistica del Rinascimento, Mantova, 2005; Gabriella ZARRI, Rosanna GOLINELLI BERTO (eds.), Osanna Andreasi da Mantova 1449-1505: tertii Praedicatorum ordinis diva, Mantova, 2006; Angela GHIRARDI, Rosanna GOLINELli BerTo (eds.), La beata Osanna e i Domenicani a Mantova: in memoria di Nicola Fiasconaro, Mantova, 2011; Angela GHIRARDI, Rosanna GOLINELLI BERTO (eds.), Osanna e Orsola: arte, storia e fede nel Seicento tra Mantova e il Monferrato, Mantova, 2018.

46 Valerio GuazZoni, La beata Stefana da Soncino nel solco di Osanna. L'immagine e il legato artistico, in Renata CASARIN (ed.), Osanna Andreasi da Mantova 1449-1505: immagine di una mistica, pp. 7997.

47 Gabriella ZARRI, Pietà e profezia alle corti padane: le pie consigliere dei principi, in Il Rinascimento alle corti padane. Società e cultura, Bari, 1977, pp. 201-237, ora in ead., Le sante vive... [ver n. 1], pp. 5185.

48 Enrico Lusso, La committenza di Anne d' Alençon. Itinerari culturali e architettonici in Monferrato al crepuscolo del marchesato paleologo, in Langhe Roero Monferrato. Cultura materiale-società-territorio, I, nr.1, 2010, pp. 19-34 (periodico on-line dell'Associazione Culturale Antonella Salvatico).

49 Su Elena Duglioli, vid. Gabriella ZARRI, Le sante vive... [ver n. 1], pp. 165-196.

50 Gabriella ZARRI, Tra mistica e Sacra Scrittura: Lucrezia Borgia e le corti femminili del primo Cinquecento, relazione presentata alla XXII Settimana di Alti Studi: L'inquieto Rinascimento di Lucrezia Borgia (Ferrara, Palazzo Bonacossi, 24-26 ottobre 2019), in Schifanoia, 60-61 (2020), pp. 51-58. 
condivise con la madre e la corte mantovana l'interesse verso le donne devote che potevano costituire una protezione per la città e un baluardo dello stato. Alla metà degli anni Trenta infatti la corte di Mantova era intenta ad ottenere il riconoscimento del culto di una donna ravennate, Margherita da Russi ${ }^{51}$, venerata da Isabella d'Este. In questo contesto si può forse comprendere il tentativo dei domenicani di riattivare l'interesse verso Maddalena da Trino componendone la leggenda agiografica.

Nel 1533 il marchesato di Monferrato si era estinto per mancanza di discendenza maschile ed era governato da Margherita Paleologo, insieme con il marito Federico II duca di Mantova. Girolamo da Milano compose la vita della terziaria trinese assumendo come modello Caterina da Siena e Osanna da Mantova. I manoscritti superstiti della leggenda agiografica, pur essendo copie, esprimono chiaramente questo intento. Nel secondo dei manoscritti sopra indicati si cita esplicitamente Osanna da Mantova come modello a cui Maddalena intendeva ispirarsi, cosa per altro molto improbabile perché le due donne erano coetanee e Osanna morì due anni dopo Maddalena. Nel manoscritto più antico invece, quello da cui ho citato, il marchese Guglielmo Paleologo viene confuso con Guglielmo Gonzaga.

La cessazione della signoria dei Paleologi sul Monferrato è ragione sufficiente per spiegare il mancato inizio della procedura di canonizzazione per la santa viva locale, che pure aveva avuto immediato culto popolare, e il tentativo di presentarla come santa di corte dei Gonzaga. L'espediente di Girolamo da Milano, tuttavia, non ebbe successo. Il culto per l'umile terziaria di Trino venne ripreso in patria nel primo Seicento per iniziativa della curia vescovile di Casale Monferrato, che il 29 maggio 1610 incaricò il Priore dei domenicani di Trino, padre Giulio Vincenzo Bertagna, di raccogliere le deposizioni dei miracoli fino allora avvenuti per intercessione di Maddalena Panatieri ${ }^{52}$. Proseguì poi con la stesura e la pubblicazione di una nuova vita della terziaria domenicana ad opera del parente fra Pietr'Antonio Fondazucca che ne scrisse la vita ${ }^{53}$ arricchendola di particolari biografici appresi in famiglia e corredandola di numerosi miracoli avvenuti nel Cinquecento e Seicento.

51 Gabriella ZARRI, Madri e maestre: Margherita Molli e Gentile Giusti nel primo Cinquecento ravennate, con una Appendice documentaria a cura di Gianni NIGRELLI, in Eraldo BALDINI e Dante BOLOGNESI (eds.), Storia di Russi. Dalla villa alla città, Ravenna 2014, pp. 499-514.

52 ASPOP-T. Trino, S. Caterina V. M., R 4. Copia replicata di tredici deposizioni di miracoli.

53 Gaspare Antonio DE GREgORY, Istoria della vercellese letteratura ed arti. Parte terza, Tipografia Chirio e Mina, Torino, 1821, pp. 126-127. 
Tra gli scrittori dell'Ordine domenicano che menzionarono Maddalena da Trino, Serafino Razzi non poté usufruire dei dati biografici forniti da Fondazucca, mentre Marchesi li utilizzò ampiamente ${ }^{54}$.

Nel borgo di Trino il ricordo di Maddalena rimase vivo nel corso del Seicento, essendovi l'usanza di celebrare solennemente la ricorrenza della sua festa, il 13 ottobre. In quel giorno si celebravano messe votive e si teneva un panegirico. La cera dell'altare veniva offerta dalla città per «pubblico antico statuto» ${ }^{55}$. Tuttavia in un anno imprecisato, tra il 1620 e il 1644, il corpo della beata venne trafugato.

Nel 1729 il vescovo di Casale, su sollecitazione del Priore domenicano di Trino, incaricò un suo delegato di raccogliere le memorie relative al culto della beata e tra il 1730 e il 1732 si tennero e registrarono le deposizioni dei miracoli. Non vi fu ancora un vero e proprio processo di canonizzazione. Nel 1729 infine il padre generale OP Thomas Ripoll chiese al Vescovo di Casale di informarsi sull'antichità del culto della Panatieri prima dei decreti di Urbano VIII ${ }^{56}$. Il processo per culto ab immemorabili venne formato nel secolo XIX e si concluse con il decreto di beatificazione, avvenuto, come si è detto, nel $1827^{57}$.

Nei secoli XIX e XX il culto locale della «Mamma» di Trino continuò ininterrotto con manifestazioni di grande rilievo, come la celebrazione del centenario della morte nel 1903, accompagnata dalla commissione di una grande statua in legno, scolpita a Monaco di Baviera ${ }^{58}$.

Nel 1964 avvenne poi un evento inaspettato di grande rilievo: si trovarono alcuni resti scheletrici nella chiesa domenicana di San Pietro Martire di Trino. Tali reperti furono sottoposti a perizia medica e riconosciuti come appartenenti alla Panatieri. Dopo apposito esame da parte delle autorità religiose, la Sacra Congregazione per le cause dei santi approvò che lo scheletro rinvenuto fosse riconosciuto come quello di Maddalena e nel 1973 l'Arcivescovo di Vercelli autorizzò i fedeli trinesi a venerare il corpo ed esporre le ossa custodite all'interno di un'urna dorata ${ }^{59}$.

54 Domenico Maria MARCHESE, Sagro diario domenicano. Tomo quinto: Nel quale si contengono le vite de' santi beati e venerabili dell'Ordine dei predicatori morti nelli due mesi settembre et ottobre, Napoli, nella stamp. di Giacinto Passaro, 1679, pp. 326-330.

55 AGOP. Roma, Santa Sabina, X1847, Istruzione sul culto della Beata Maddalena da Trino del III Ordine di San Domenico.

56 Ibidem.

57 Ibid., X1848 e X1849.

58 Franco Crosio, Una sostanza che non muta (1903-2003), in GIROlamo DA Milano, La beata Maddalena da Trino. Maddalena donna di Dio... [ver n. 20], pp. 113-121.

59 Renato VASCONI, La donna che incarnò la gioia... [ver n. 18], pp. 97 e segg. 
Le ossa e le altre reliquie della beata vennero custodite nella chiesa dei domenicani di Trino, dove erano rimaste per secoli, ma nel 1995 la Provincia domenicana decise di chiudere il convento di Trino. A quella data la custodia delle reliquie passò alla Fraternita laica domenicana ${ }^{60}$, che continuò a curare le celebrazioni annuali della festa ed altre manifestazioni mensili, fino all'organizzazione del quinto Centenario della morte della beata, nel 2003, che fu l'occasione per far conoscere anche ai ragazzi delle scuole elementari la vita della loro antica protettrice e per raccogliere in una pubblicazione testimonianze fotografiche di dipinti, immagini ed ex voto che provano la continuità del culto locale della terziaria trinese ${ }^{61}$.

Ignorato l'antico rapporto che Maddalena aveva con il dimenticato Marchese Paleologo, i fedeli di Trino dovevano continuare per secoli a confidare nella loro protettrice, in cui vedevano forse una madre generosa disposta a sacrificarsi per la città e a elargire grazie a chi la invocava.

\section{BIBLIOGRAFIA}

Aletto, Carlo, Perin, Antonella (eds.), Casale Monferrato, una capitale per il territorio: le premesse: da Teodoro 2. a Giovanni 4. (1404-1464): atti del Convegno, Genova, 2019

ArCangeli, Letizia, GentILE, Marco (eds.), Le signorie dei Rossi di Parma tra XIV e XVI secolo, Firenze, 2007.

BaldinI, Eraldo, Bolognesi, Dante (eds.), Storia di Russi. Dalla villa alla città, Ravenna 2014.

Bartolomei Romagnoli, Alessandra, Santità e mistica femminile nel Medioevo, Spoleto, 2013.

BARTOLOMEI ROMAGNOLI, Alessandra, Caterina e Robina: profezia e antiprofezia al tempo del grande scisma d'Occidente, in EAD., Santità e mistica femminile nel Medioevo, Spoleto, 2013, pp. 630-656.

Bartolomei Romagnoli, Alessandra, Cinelli, Luciano, Piatti, Pierantonio (eds.), Virgo digna coelo: Caterina e la sua eredità: raccolta di studi in occasione del $550^{\circ}$ anniversario della canonizzazione di santa Caterina da Siena (1461-2011), Città del Vaticano, Libreria Editrice Vaticana, 2013.

60 Cfr. Girolamo da Milano, La beata Maddalena da Trino. Maddalena donna di Dio... [ver n. 20], p. 52.

61 Ibid., pp. 59-108 dove sono pubblicati gli elaborati sulla beata dell'anno scolastico 2003-2004 di alcune scuole elementari e medie. Il volume riporta anche le immagini fotografiche di reliquie e santini della beata, oltre alla fotografia di ex voto e tavolette votive dipinte. 
Bartolomei Romagnoli, Alessandra, Cinelli, Luciano, Piatti, Pierantonio (eds.), Angeliche visioni: Veronica da Binasco nella Milano del Rinascimento, Firenze, Edizioni del Galluzzo per la Fondazione Ezio FranCESCHINI, 2016.

Bartolomei Romagnoli, Alessandra (ed.), Sante vive in Europa (secoli XV-XVI)/Santas vivas en Europa (siglos $X V$-XVI), numero monografico di Archivio italiano per la storia della pietà, XXXIII, 2020.

Battioni, Gianluca, Aspetti della politica ecclesiastica di Pier Maria Rossi, in Letizia ARCANGELI, Marco GENTILE (eds.), Le signorie dei Rossi di Parma tra XIV e XVI secolo, Firenze, 2007, pp. 106-107.

BianCA, Concetta, SCATTIGNO, Anna (eds.), Scritture carismi istituzioni: percorsi di vita religiosa in età moderna. Studi per Gabriella Zarri, Roma, 2018.

Blumenfeld-Kosinski, Renate, Venarde, Bruce L., Two Women of the Great Schism: The Revelations of Constance de Rabastens by Raymond de Sabanac and the Life of Ursulina of Parma by Simone Zanacchi, Toronto, 2010.

Blumenfeld-Kosinski, Renate, Poets, Saints, and Visionaries of the Great Schism (13781417), University Park, Pennsylvania 2006.

BornsteIn, Daniel, RUSCONI, Roberto (eds.), Mistiche e devote nell'Italia tardomedievale, Napoli, 1992.

BOESCH GAJANO, Sofia, Le «sante vive»: un'invenzione storiografica da brevettare, in BIANCA, Concetta, SCATTIGNO, Anna (eds.), Scritture carismi istituzioni: percorsi di vita religiosa in età moderna. Studi per Gabriella Zarri, Roma, 2018, pp. 361-366.

BuganZA, Stefania, RAININI, Marco Giuseppe (eds.), Il convento di Santa Maria delle Grazie a Milano. Una storia dalla fondazione a metà del Cinquecento. Atti del Convegno di Studi (Milano, 22-24 maggio 2014), (Memorie domenicane, n. s. 47 (2016).

Catálogo de Santas Vivas (1400-1550): Hacia un corpus completo de un modelo bagiográfico femenino diretto da Rebeca SANMARTÍN BASTIDA http://catalogodesantasvivas.visionarias.es/index.php/P\%C3\%A1gina_principal\#Proyecto [consultato il 15 marzo 2021].

CASARIN, Renata (ed.), Osanna Andreasi da Mantova 1449-1505: immagine di una mistica del Rinascimento, Mantova, 2005.

Cereghino op, Matteo, La Beata Maddalena Panatieri da Trino, Genova, 1927.

CONTAMIne, Philippe, Bouzy, Olivier, HÉLARY, Xavier, Jeanne d'Arc: bistoire et dictionnaire, Paris, Laffont, 2012.

CONTAMine, Philippe, De feanne d'Arc aux guerres d'Italie : figures, images et problèmes du $X V^{e}$ siècle, Orléans, Paradigme, 1994.

Corrain, Lucia, Di TeOdoro, Francesco P. (eds.), Architettura e identità locali, vol. I; indici a cura di Emanuela VAI, Firenze, 2013.

Crosio, Franco, Una sostanza che non muta (1903-2003), in GIROLAMO DA MilaNO, La beata Maddalena da Trino. Maddalena donna di Dio, donna del Rinascimento, Trino, Tipografia AGS, 2005, pp. 113-121.

DEgL'InNOCEnTI, Antonella, Un'opera da riscoprire: le Visiones della beata Osolina da Parma, in Hagiographica, XXVII, 2020, pp. 379-402. 
DÓCI OP, Viliam Štefan, FESTA OP, Gianni (eds.), Fra trionfi e sconfitte. La «politica della santità» dell'Ordine dei Predicatori, Roma, 2021.

FondazuCCA, P., Vita della beata Maddalena Pannatieri da Trino Professa del Terz'Ordine del Patriarca S. Domenico... Dedicata a' molt' illustri signori Sindici di Trino, Milano, Francesco Vigone, 1702 (I ediz. 1644, come si deduce dalla lettera dedicatoria datata 1644).

GHIRARDI, Angela, GOLINELli BERTO, Rosanna (eds.), La beata Osanna e i Domenicani a Mantova: in memoria di Nicola Fiasconaro, Mantova, 2011.

GHIRARDI, Angela, GOLINELli BERTO, Rosanna (eds.), Osanna e Orsola: arte, storia e fede nel Seicento tra Mantova e il Monferrato, Mantova, 2018.

Girolamo Da Milano, La beata Maddalena da Trino. Maddalena donna di Dio, donna del Rinascimento, Trino, Tipografia AGS, 2005.

GuazZONI, Valerio, La beata Stefana da Soncino nel solco di Osanna. L'immagine e il legato artistico, in Renata CASARIN (ed.), Osanna Andreasi da Mantova 1449-1505: immagine di una mistica, pp. 79-97.

HERZIG, Tamar, Savonarola's women: visions and reform in renaissance Italy, Chicago; London, 2008.

ISZAK, Angelico, Panatieri, Maddalena, beata, in Bibliotheca Sanctorum, vol. 10, Roma, 1998, coll. 77-78.

ISOLANI, Isidoro, Inexplicabilis mysterii gesta beatae Veronicae virginis praeclarissimi monasterii sanctae Marthae urbis Mediolani. Sub obseruatione regulae diui Augustini, Mediolani, apud Gotardum Ponticum impressorum, 1518 die III Aprilis.

Acta sanctorum, Auctarium ad diem XIV Octobris, De B. Magdalena de Panateriis Virgine, Tertii Ordinis S. Dominici, Tridini in Dioecesi Vercellensi, Bruxelles, 1965-1970 (Ripr. facs. dell'ed.: Anversa, poi Bruxelles, 1643-1940), pp. 168-178.

LANZARDO, Diego, TARICCO, Bruno (eds.), Gli ordini mendicanti e la città. I frati predicatori, Cherasco, 2009 ( $\ll$ Miscellanea di Storia degli insediamenti», I).

Lusso, Enrico, La committenza architettonica dei Marchesi di Saluzzo e di Monferrato nel tardo Quattrocento: modelli mentali e orientamenti culturali, in CORRAIN, Lucia, DI TEODORO, Francesco P. (eds.), Architettura e identità locali, vol. I; indici a cura di Emanuela VAI, Firenze, 2013, pp. 423-438.

Lusso, Enrico, I conventi del principe. Fondazioni dei Predicatori e strategie urbane nel Monferrato paleologo, in Gli ordini mendicanti e la citta'. I frati predicatori, a cura di LANZARDO, Diego, TARICCO, Bruno (eds.), Cherasco, 2009 ( $\ll$ Miscellanea di Storia degli insediamenti», I), pp. 89-120: 89-97.

Lusso, Enrico, La committenza di Anne d'Alençon. Itinerari culturali e architettonici in Monferrato al crepuscolo del marchesato paleologo, in Langhe Roero Monferrato. Cultura materiale-società-territorio, I, nr.1, 2010, pp. 19-34 (periodico on-line dell'Associazione Culturale Antonella Salvatico).

Marchese, Domenico Maria, Sagro diario domenicano. Tomo quinto: Nel quale si contengono le vite de' santi beati e venerabili dell'Ordine dei predicatori morti nelli due 
mesi settembre et ottobre, Napoli, nella stamp. di Giacinto Passaro, 1679, pp. 326330.

MATter, E. Ann, ZARRI, Gabriella, Una mistica contestata. La Vita di Lucia da Narni (1476-1544) tra agiografia e autobiografia. Con l'edizione del testo, Roma, Edizioni di Storia e Letteratura, 2011.

NocentinI, Silvia, Le Grazie nell'opera di Ambrogio Taegio in BuganZA, Stefania, RAININI, Marco Giuseppe (eds.), Il convento di Santa Maria delle Grazie a Milano. Una storia dalla fondazione a metà del Cinquecento. Atti del Convegno di Studi (Milano, 2224 maggio 2014), (Memorie domenicane, n. s. 47 (2016), pp. 79-102.

Oen, Maria H. (ed.), A companion to Birgitta of Sweden and her Legacy in the Later Middle Ages, Leiden; Boston, Brill, 2019.

OEN, Maria H., FALKEID, Unn (eds.), Sanctity and female authorship: Birgitta of Sweden d Catherine of Siena, New York; London, Routledge, 2020.

ParaVicini Bagliani, Agostino, VAUCHEZ, André (eds.), Poteri carismatici e poteri informali, Palermo, 1992.

PERIN, Antonella, SOLARINO, Carla, Casale e il territorio nella prima metà del secolo XV: architetture tra perdite e sopravvivenze, in Carlo AleTto, Antonella PERIN (eds.), Casale Monferrato, una capitale per il territorio: le premesse: da Teodoro 2. a Giovanni 4. (1404-1464): atti del Convegno, Genova, 2019.

PIERRE, Benoist, VAUCHEZ, André (eds.), L'eremita Francesco di Paola viandante e penitente. Atti del III Convegno internazionale di studio (Paola, 14-16 settembre 2000), Roma, 2006.

Pierre, Benoist, Vauchez, André (eds.), Saint Francois de Paule et les Minimes en France de la fin $d u X V^{e}$ au XVIII siècle, textes réunis et présentés, Tours, 2010.

PIERRE, Benoist, VAUCHEZ, André (eds.), Commende, Osservanza e riforma tra Italia, Francia e Spagna. Atti del Convegno di studi (Roma, 22-24 novembre 2007), Roma, 2014.

RAZZI, Serafino, Vite de i santi, e beati del sacro Ordine de' frati predicatori, cosi buomini, come donne. Con aggiunta di molte vite, che nella prima impressione non erono. In Firenze, nella stamperia di Bartolomeo Sermartelli, 1588.

Romano, Giovanni, Cane, Ottaviano, in Dizionario biografico degli italiani (=DBI), vol. 17 (1974), https://www.treccani.it/enciclopedia/ottaviano-cane_(Dizionario-Biografico)/

Rosso, Paolo, La cultura delle èlites marchionali nella Casale della prima metà del Quattrocento, in AletTo, Carlo, PerIN, Antonella (eds.), Casale Monferrato, una capitale per il territorio: le premesse: da Teodoro 2. a Giovanni 4. (1404-1464): atti del Convegno, Genova, 2019, pp. 31-69.

Rosso, Paolo, La politica culturale dei Paleologi fra Quattro e Cinquecento e i suoi riflessi nell'editoria del marchesato, in BALBONI, Magda (ed.), Trino e l'arte tipografica nel XVI secolo, Novara, Interlinea, 2014, pp. 71-90.

SETTIA, Aldo, Guglielmo VIII, Marchese del Monferrato, in DBI, vol. 60, 2003, https://www. treccani.it/enciclopedia/guglielmo-viii-marchese-di-monferrato_\%28Dizionario-Biografico\%29/ 
SignORINI, Rodolfo, Golinelli BerTo, Rosanna (eds.), Osanna Andreasi da Mantova 1449-1505: la santità nel quotidiano, Mantova, 2005.

SILVESTRI, Francesco, Beatae Osannae Mantuanae de tertio babitu ordinis fratrum predicatorum uita per fratrem Franciscum Siluestrum Ferrariensem eiusdem ordinis \& uitae regularis professorem edita, Impressum Mediolani, per Petrum Martirem de Mantegatiis dictum Cassanum, 1506 die 20 Augusti.

SILVESTRI, Francesco, La vita e stupendi Miraculi dela gloriosa virgine Osanna Mantuana del Terzo ordine de Frati Predicatori (Impressa in Milano, apresso di Alexandro Minutiano, a sexdeci di Ianuario, 1507).

Sacra Rituum Congregatione E.mo et R.mo Domino Cardinali de Somalia Praefecto et ponente, Tridentinen, seu Ordinis Praedicatorum pro confirmatione cultus ab immemorabili tempore praestiti B. Magdalenae de Panateriis Tertii Ordinis S: Dominici... Memoriale cum Summario, Animadversiones R.P.D. Promotoris Fidei, et Responsiones, Romae MDCCCXXVII, ex Typographia Reverendae Camerae Apostolicae.

VASCONI, Renato, La donna che incarnò la gioia: storia della beata Maddalena da Trino, Alba, 1972.

VAUCHEZ, André, Saints, prophètes et visionnaires: le pouvoir surnaturel au Moyen Age, Paris, Albin Michel, 1999.

ZANICHELLI, Giuseppa Z., La committenza dei Rossi: immagini di potere fra sacro e profano, in ARCANgeli, Letizia, Gentile, Marco (eds.), Le signorie dei Rossi di Parma tra XIV e XVI secolo, Firenze, 2007, p. 211.

ZARRI, Gabriella, Le sante vive. Per una tipologia della santità femminile nel primo Cinquecento, in Annali dell'Istituto Storico ItaloGermanico in Trento, 6 (1980), pp. 372-445, ora in EAD., Le sante vive. Cultura e religiosità femminile nella prima età moderna, Torino 1990.

ZARRI, Gabriella, Les prophètes de cour dans l'Italie de la Renaissance, in «Mélanges de l'Ecole Française de Rome - Moyen Age», Tome 102, 1990 - 2, pp. 649-675.

ZARRI, Gabriella, Potere carismatico e potere politico nelle corti italiane del Rinascimento, in Bagliani, Agostino, Vauchez, André (eds.), Poteri carismatici e poteri informali, Palermo, 1992, pp. 164180.

ZARRI, Gabriella, Profeti di corte nell'Italia del Rinascimento, in BORNSTEIN, Daniel, RUSCONI, Roberto (eds.), Mistiche e devote nell'Italia tardomedievale, Napoli, 1992, pp. 209-236, ora in EAD., La chiesa dei principi e delle città tra medioevo e prima età moderna, Spoleto, 2021, pp. 267-294.

ZARRI, Gabriella, GOlinelli BerTo, Rosanna (eds.), Osanna Andreasi da Mantova 14491505: tertii Praedicatorum ordinis diva, Mantova, 2006.

ZARRI, Gabriella, Madri e maestre: Margherita Molli e Gentile Giusti nel primo Cinquecento ravennate, con una Appendice documentaria a cura di NigRELLI, Gianni, in BALDINI, Eraldo, Bolognesi, Dante (eds.), Storia di Russi. Dalla villa alla città, Ravenna 2014, pp. 499-514.

ZARRI, Gabriella, La chiesa dei principi e delle città tra medioevo e prima età moderna, Spoleto, 2021. 
ZARRI, Gabriella, Tra mistica e Sacra Scrittura: Lucrezia Borgia e le corti femminili del primo Cinquecento, relazione presentata alla XXII Settimana di Alti Studi: L'inquieto Rinascimento di Lucrezia Borgia (Ferrara, Palazzo Bonacossi, 24-26 ottobre 2019), in Schifanoia (2020), pp. 60-61.

ZARRI, Gabriella, Legittimare il principe: principi rinascimentali italiani e la promozione del culto dei propri santi domenicani, in DóCI OP, Viliam Štefan, FESTA OP, Gianni (eds.), Fra trionfi e sconfitte. La «politica della santità» dell'Ordine dei Predicatori, Roma, 2021, in corso di stampa. 


\section{HISTORIOGRAFÍA Y BIBLIOGRAFÍA}


